\title{
Explicit Birefringence Analysis for Anisotropic Fibers
}

\author{
Ruey-Beei Wu
}

\begin{abstract}
A hyper-perturbation approach is employed to find the Taylor expansion formulas for the dispersion curves of arbitrarily indexed optical fibers. These formulas are then used to give analytically the birefringence characteristics of optical fibers with biaxially anisotropic core and cladding. The dependence of the fiber birefringence on the index difference, index anisotropy, and the normalized frequency is explicitly determined. Simple, accurate analytic formulas have been presented for anisotropic fibers with step- and parabolic-index profiles. Numerical results are in good agreement with the literature results, whenever available.
\end{abstract}

\section{INTRODUCTION}

$\mathbf{I}$ $\mathrm{N}$ reality, all conventional single-mode fibers support two orthogonal polarizations. Bends and twists, unavoidable for the practical fibers, have the property of coupling energy between these two degenerate modes. For sophisticated systems like coherent communication systems, sensory systems based on interferometric principles, or systems utilizing polarization-dependent devices, these changes in the state of polarization would lead to signal fading and noise. To overcome this, isotropic fibers with noncircular geometry and fibers with anisotropic material have been proposed to remove the polarization degeneracy and reduce the polarization mode conversion [1]. Most of the high birefringence fibers have a built-in stress-applying components which makes the core and/or cladding biaxially anisotropic.

Several sophisticated numerical methods have been proposed to investigate the birefringence characteristics of anisotropic optical fibers. Under the weakly guiding assumption, Lindell and Oksanen proposed a variational analysis for step- [2] and parabolic- [3] index fibers, which relies much on the choice of trial functions and still suffers from worse accuracy for fibers operating in near cutoff or well-guided region. Wu and Chen presented a general variational reaction analysis [4] which is applicable for arbitrarily indexed fibers even when the index difference is large, in the price of a huge computational load. Recently, Chen derived a very tedious perturbation analysis [5] which is applicable for step-index fibers only and cannot yield good results in the near cutoff region.

In this paper, we present a novel hyper-perturbation approach to find the Taylor expansion of the dispersion relation

Manuscript received February 21, 1991; revised May 16, 1991. This work was supported in part by the National Science Council, Republic of China, under Grant NSC-79-0417-E002-09.

The author is with the Department of Electrical Engineering, National Taiwan University, Taipei, Taiwan, ROC

IEEE Log Number 9103846. such that the propagation constant can be explicitly expressed by the power series of frequency. First of all, the conventional finite element method or the variational reaction theory [6] is employed to solve the propagation constant and modal field of an arbitrarily indexed optical fibers. The expansion coefficients up to very high orders can then be calculated directly via a few simple matrix manipulations once the eigen-solution is solved at a certain frequency. The whole dispersion curve can be approximated to a high accuracy by the expansion formulas at few distinct frequencies. Given the explicit expressions for propagation constants, it becomes straightforward to determine the birefringence characteristics for weakly guiding fibers with anisotropic core and/or cladding.

\section{Hyper-Perturbation Theory}

Consider an optical fiber with the refractive-index profile

$$
\begin{aligned}
n^{2}(x) & =n_{d}^{2}+\left(n_{r}^{2}-n_{d}^{2}\right) \cdot f(R) & & \text { for } R=r / a \leq 1 \\
& =n_{d}^{2} & & \text { for } r>a
\end{aligned}
$$

where $a$ is the core radius, $n_{d}$ is the cladding index, $n_{r}$ is the index maximum in the core, and function $f(R)$ defines the profile shape. As the index difference tends to zero, it is well known that the propagation characteristics can be determined from the $\mathrm{LP}_{\mathrm{mn}}$ mode which satisfies the scalar-wave equation

$$
\frac{1}{R} \frac{d}{d R}\left(R \frac{d \psi}{d R}\right)+\left[V^{2} \cdot f(R)-W^{2}-\left(\frac{m}{R}\right)^{2}\right] \psi=0
$$

under the boundary condition

$$
\left.\frac{1}{\psi} \frac{d \psi}{d R}\right|_{R=1} \equiv Z_{m}(W)=\frac{W \cdot K_{m}^{\prime}(W)}{K_{m}(W)} .
$$

Here, the normalized frequency $V$ and the parameter $W$ are defined by

$$
V=k a \sqrt{n_{r}^{2}-n_{d}^{2}} \text { and } W=a \sqrt{\beta^{2}-n_{d}^{2} \cdot k^{2}}
$$

respectively, $K_{m}(W)$ is the $m$ th order modified Bessel function of the second kind, and the prime ' denotes the differentiation with respect to the argument $W$.

Consider the integration of (2) multiplied with an arbitrary test function $\psi^{a}$. By employing the integration by part and imposing the boundary condition, we can obtain a reaction formula for the guided mode [6]

$$
\left\langle\psi^{a}|\mathcal{H}| \psi\right\rangle=\int_{0}^{1}\left\{\left[V^{2} f-W^{2}-\left(\frac{m}{R}\right)^{2}\right] \psi^{a} \cdot \psi\right.
$$




$$
\begin{aligned}
\left.-\frac{d \psi^{a}}{d R} \cdot \frac{d \psi}{d R}\right\} & R d R \\
& +Z_{m}(W) \cdot \psi^{a}(1) \cdot \psi(1)
\end{aligned}
$$

Equation (3) will be zero for arbitrary field $\psi^{a}$ if $W$ and $\psi$ are the desired eigen-solution. By choosing suitable mesh division and basis functions in the finite element method, (3) can be reduced to a matrix equation. Given a frequency $V_{0}$, we can search for zero determinant of the matrix, [7, ch. 11] to locate the associated eigenvalue $W_{0}$ and solve the modal field $\psi_{0}$. In general, the determinant is a nonlinear function of $W$ and $V$ such that the eigen-search process has to be repeated each time the frequency is changed.

Conversely, we can treat the frequency $V$ as a perturbation and expand the eigenvalue $W$ and modal field $\psi$ near the frequency $V_{0}$ by

$$
\begin{gathered}
W=W_{0}+\sum_{n=1}^{\infty} W_{n} \cdot\left(V-V_{0}\right)^{n} \\
\psi=\psi_{0}+\sum_{n=1}^{\infty} \psi_{n} \cdot\left(V-V_{0}\right)^{n}
\end{gathered}
$$

where

$$
W_{n}=\left.\frac{1}{n !} \frac{d^{n} W}{d V^{n}}\right|_{V=V_{0}} \quad \text { and } \quad \psi_{n}=\left.\frac{1}{n !} \frac{d^{n} \psi}{d V^{n}}\right|_{V=V_{0}}
$$

are the expansion coefficient and the expansion field, respectively. Since the operator $\mathcal{H}$ in (3) is dependent on $V$ as well as $W$, it can also be expanded as

$$
\mathcal{H}(V, W)=\mathcal{H}_{0}+\sum_{n=1}^{\infty} \mathcal{H}_{n} \cdot\left(V-V_{0}\right)^{n}
$$

where $\mathcal{H}_{0}=\mathcal{H}\left(V_{0}, W_{0}\right)$ and the expansion operator $\mathcal{H}_{n}$ is defined by

$$
\mathcal{H}_{n}=\left.\frac{1}{n !} \frac{d^{n} \mathcal{H}}{d V^{n}}\right|_{V=V_{0}, W=W_{0}} \equiv \mathcal{H}_{n v}+\frac{\partial \mathcal{H}}{\partial W} W_{n}
$$

It should be noted that the operator $\mathcal{H}_{n v}$ depends on $V_{0}$, $W_{0}$, and the expansion coefficients $W_{1}, W_{2}, \cdots$, up to $W_{n-1}$ but not $W_{n}$. For example, it equals $\partial \mathcal{H} / \partial V$ as $n=1$ and $\frac{1}{2} \partial^{2} \mathcal{H} / \partial V^{2}+W_{1} \cdot \partial^{2} \mathcal{H} / \partial V \partial W$ as $n=2$. Since the operator $\mathcal{H}$ defined in (3) is expressed explicitly in terms of $V$ and $W$, the derivatives in (7) can be taken analytically.

By substituting (4)-(6) into the reaction formula (3) and neglecting all the terms with second power of $\left(V-V_{0}\right)$ or higher, we obtain the first order formula

$$
\left\langle\psi^{a}\left|\mathcal{H}_{0}\right| \psi_{1}\right\rangle=-\left\langle\psi^{a}\left|\mathcal{H}_{1}\right| \psi_{0}\right\rangle
$$

If the arbitrary field $\psi^{a}$ in (8) is replaced by the modal field $\psi_{0}$, it is not difficult to prove that the left-hand side (LHS) in (8) is zero. Then, the first-order derivative of $W$, i.e., $W_{1}$, can be found by

$$
W_{1}=-\left\langle\psi_{0}\left|\frac{\partial \mathcal{H}}{\partial V}\right| \psi_{0}\right\rangle /\left\langle\psi_{0}\left|\frac{\partial \mathcal{H}}{\partial W}\right| \psi_{0}\right\rangle
$$

Once the eigen-solution $\left(W_{0}, \psi_{0}\right)$ is obtained, the terms in (9) can be calculated by numerical integration. In this way, the derivative $W_{1}$ is determined without taking any numerical differentiation. Equation (9) is nothing more than the conventional formula given in the Rayleigh quotient [8] or the first-order perturbation theory [9].

Given the eigen-solution $\left(W_{0}, \psi_{0}\right)$ as well as $W_{1}$, the operator $\mathcal{H}_{1}$ as defined in (7) is available now. Since $(8)$ is valid for arbitrary field $\psi^{a}$, it can be employed to find the first-order expansion field $\psi_{1}$. By the finite element method choosing the same mesh division and basis functions, (8) becomes a matrix equation for the nodal unknowns corresponding to $\psi_{1}$. Here, the matrix corresponding to the operator $\mathcal{H}_{0}$ is already available, while the right-hand side (RHS) vector is contributed by the term $\left\langle\psi^{a}\left|\mathcal{H}_{1}\right| \psi_{0}\right\rangle$. It should be noted that the matrix is singular at the eigen-solution and the modal field $\psi_{0}$ is determined up to an arbitrary constant. Hence, an additional boundary condition is required to solve the matrix equation for $\psi_{1}$ uniquely. A simple way is to choose that one of the nodal values, usually the boundary one, of the modal field $\psi_{0}$ is a constant. Then, we achieve an essential boundary condition for (8), e.g., $\psi_{1}$ is enforced to be zero at that node.

Similar process can be repeated to calculate the higher order expansion coefficients. Substituting (4)-(6) into the reaction formula (3) and equating the terms with power $\left(V-V_{0}\right)^{n}$, we now obtain the following recursive relation:

$$
\left\langle\psi^{a}\left|\mathcal{H}_{0}\right| \psi_{n}\right\rangle=-\sum_{i=1}^{n}\left\langle\psi^{a}\left|\mathcal{H}_{i}\right| \psi_{n-i}\right\rangle
$$

which holds for arbitrary field $\psi^{a}$. If $\psi^{a}$ is chosen as the modal field $\psi_{0}$, we can still show that the LHS of (10) is zero. Hence, the expansion coefficient $W_{n}$ can be found by

$$
\begin{aligned}
W_{n}= & -\left\{\left\langle\psi_{0}\left|\mathcal{H}_{n v}\right| \psi_{0}\right\rangle+\sum_{i=1}^{n-1}\left\langle\psi_{0}\left|\mathcal{H}_{i}\right| \psi_{n-i}\right\rangle\right\} \\
& /\left\langle\psi_{0}\left|\frac{\partial \mathcal{H}}{\partial W}\right| \psi_{0}\right\rangle .
\end{aligned}
$$

On the other hand, (10) can be employed to solve the $n$th order expansion field $\psi_{n}$ from the lower order expansions $W_{i}$ and $\psi_{i}(i=0,1, \cdots, n-1)$ as well as the just solved $W_{n}$. Again, the matrix representation for $\mathcal{H}_{0}$ is singular and the essential boundary condition requires that $\psi_{n}=0$ at a particular node.

\section{EXPLICIT DISPERSION FORMULAS}

Based on the hyper-perturbation theory together with the finite element method, a Fortran program is tailored on IBM/PC to solve the various order expansion coefficients for guided LP modes of optical fibers. The solution region is uniformly divided into $M$ elements, inside each of which the quadratic basis is employed. Since the index profile may be of arbitrary shape $f(R)$, the element integral is evaluated by the four-point Gaussian Legendre quadrature. Given an operating frequency $V_{0}$, the program first searches for the eigenvalue $W_{0}$ and then calculates the Taylor expansion coefficients of the dispersion curve up to an arbitrary order.

Since the algorithm involves numerical integration instead of differentiation, the calculated expansion coefficients $W_{n}$ converge very fast as $M$ increases. Numerical analysis shows 
that $M=5$ is enough to make $W_{n}$ converge with relative error smaller than $10^{-4}$ for step-index fibers. More divisions are required for graded index fibers. For example, $M=7$ should be chosen to achieve a similar accuracy for parabolic-index fibers.

Given the profile shape, the dispersion characteristics for guided LP modes can be uniquely determined from the parameter $W$ versus the normalized frequency $V$, say $W=$ $F(V)$. Listed here are the explicit expressions of the dispersion relations of fundamental LP modes for step- and parabolicindex fibers in the usual frequency range $0 \leq V \leq 10$. The absolute error of the results given by the following formulas is smaller than $10^{-4}$.

\section{A. Step-Index Fibers}

$$
\begin{aligned}
& W \cong 0 . \quad \text { for } 0 \leq V \leq 0.45 \\
& W=0.0004848+0.015520 y+0.20189 y^{2}+1.2852 y^{3} \\
& +3.2657 y^{4}-4.6273 y^{5}-32.642 y^{6}+43.885 y^{7} \\
& +265.40 y^{8} \\
& y \equiv V-0.5 \text { for } 0.45 \leq V \leq 0.6 \\
& W=0.0244853+0.28626 y+1.0647 y^{2}+0.58050 y^{3} \\
& -3.3021 y^{4}+1.3074 y^{5}+12.072 y^{6}-25.746 y^{7} \\
& -5.7815 y^{8 ;} \\
& y \equiv V-0.7 \text { for } 0.6 \leq V \leq 0.8 \\
& W=0.202365+0.84859 y+0.63048 y^{2}-0.73150 y^{3} \\
& +0.37318 y^{4}+0.47363 y^{5}-1.5775 y^{6}+2.2912 y^{7} \\
& -1.6084 y^{8} \\
& y \equiv V-1 \quad \text { for } 0.8 \leq V \leq 1.2 \\
& W=0.718197+1.12619 y+0.078285 y^{2}-0.12854 y^{3} \\
& +0.12172 y^{4}-0.093802 y^{5}+0.060164 y^{6} \\
& -0.028270 y^{7}+0.0019594 y^{8} \\
& y \equiv V-1.5 \text { for } 1.2 \leq V \leq 1.7 \\
& W=1.290213+1.14819 y-0.0085133 y^{2}-0.019350 y^{3} \\
& +0.019055 y^{4}-0.013432 y^{5}+0.0083050 y^{6} \\
& -0.0047200 y^{7}+0.0024839 y^{8} \text {; } \\
& y \equiv V-2 \text { for } 1.7 \leq V \leq 2.4 \\
& W=2.972755+1.09426 y-0.015769 y^{2}+0.0021716 y^{3} \\
& -0.10373 \times 10^{-3} y^{4}-0.91300 \times 10^{-4} y^{5}+0.55821 \\
& \times 10^{-4} y^{6}-0.23555 \times 10^{-4} y^{7}+0.87318 \times 10^{-5} y^{8} \text {; } \\
& y \equiv V-3.5 \text { for } 2.4 \leq V \leq 4.5 \\
& W=6.677591+1.03597 y-0.0040003 y^{2}+0.44097 \\
& \times 10^{-3} y^{3}-0.47791 \times 10^{-4} y^{4}+0.50237 \times 10^{-5} y^{5} \\
& -0.50031 \times 10^{-6} y^{6}+0.44664 \times 10^{-7} y^{7} \\
& -0.30529 \times 10^{-8} y^{8} \text {; } \\
& y \equiv V-7 \text { for } 4.5 \leq V \leq 10 \text {. }
\end{aligned}
$$

\section{B. Parabolic-Index Fibers}

$$
\begin{aligned}
& W \cong 0 . \quad \text { for } 0 \leq V \leq 0.55 \\
& W=0005082+0.011860 y+0.11299 y^{2}+0.53227 y^{3} \\
& +1.0427 y^{4}-0.78234 y^{5}-5.2432 y^{6} \\
& +3.5557 y^{7}+23.234 y^{8} \text {; } \\
& y \equiv V-0.7 \text { for } 0.55 \leq V \leq 0.8 \\
& W=0.0328590+0.26385 y+0.66751 y^{2}+0.21966 y^{3} \\
& -0.99872 y^{4}+0.38446 y^{5}+1.6633 y^{6} \\
& -2.8108 y^{7}+0.10073 y^{8} \\
& y \equiv V-1 \quad \text { for } 0.8 \leq V \leq 1.2 \\
& W=0.318137+0.80983 y+0.32464 y^{2}-0.27216 y^{3} \\
& +0.13437 y^{4}+0.013353 y^{5}-0.12003 y^{6} \\
& +0.15788 y^{7}-0.12466 y^{8} \text {; } \\
& y \equiv V-1.5 \quad \text { for } 1.2 \leq V \leq 1.8 \\
& W=1.28718+1.03570 y+0.019159 y^{2}-0.021734 y^{3} \\
& +0.013645 y^{4}-0.0070347 y^{5}+0.0032285 y^{6} \\
& +0.0013322 y^{7}+0.47687 \times 10^{-3} y^{8} \text {; } \\
& y \equiv V-2.5 \quad \text { for } 1.8 \leq V \leq 3.1 \\
& W=2.84847+1.03590 y-0.0054901 y^{2}-0.22435 \\
& \times 10^{-4} y^{3}+.39110 \times 10^{-3} y^{4}-0.18864 \times 10^{-3} y^{5} \\
& +0.67623 \times 10^{-4} y^{6}-0.21548 \times 10^{-4} y^{7} \\
& +0.64511 \times 10^{-5} y^{8} \text {; } \\
& y \equiv V-4.0 \text { for } 3.1 \leq V \leq 5 \\
& W=5.91672+1.01344 y-0.0020187 y^{2}+0.26653 \\
& \times 10^{-3} y^{3}-0.28924 \times 10^{-4} y^{4}+0.21441 \times 10^{-5} y^{5} \\
& +0.20382 \times 10^{-7} y^{6}-0.48528 \times 10^{-7} y^{7} \\
& +.12792 \times 10^{-7} y^{8} \text {; } \\
& y \equiv V-7 \text { for } 5 \leq V \leq 10 \text {. }
\end{aligned}
$$

\section{Direct Birefringence ANALysis}

Without loss of generality, consider an optical fiber with the permittivity tensor

$$
[\epsilon]=\left[\begin{array}{ccc}
n_{x}^{2} & 0 & 0 \\
0 & n_{y}^{2} & 0 \\
0 & 0 & n_{z}^{2}
\end{array}\right]
$$

where

$$
\begin{aligned}
n_{i}^{2} & =n_{d i}^{2}+\left(n_{r i}^{2}-n_{d i}^{2}\right) \cdot f_{i}(R) \\
& =n_{d i}^{2} \cdot\left[1+\Delta_{i} \cdot f_{i}(R)\right]
\end{aligned}
$$

for $i=x, y, z$. Here, $f_{i}(R)$ defines the profile shape in the principal axes. It has the maximum of unity in the core and is zero in the cladding. In practical applications, the profile anisotropy can be neglected by assuming $f_{i}(R)=f(R)$. In terms of the permittivity model (12), the cladding as well as 
core can be anisotropic, while the index profile can be step, parabolic, or arbitrary.

Under the weakly guiding assumption, usually made for optical fibers, the LP modes are linearly polarized such that only the permittivity in the polarization axis is relevant. The dispersion curve $W=F(V)$ will be identical to that for isotropic fibers, if $W$ and $V$ are now replaced by

$$
W_{i}=a \sqrt{\beta_{i}^{2}-n_{d i}^{2} \cdot k^{2}}, \quad V_{i}=k a \sqrt{n_{r i}^{2}-n_{d i}^{2}}
$$

where $i=x$ and $y$ for LP modes polarized in $x$ and $y$ directions, respectively. The function $F$ is dependent on the profile shape $f(R)$ only. Its explicit expression has been given in the above session for step and parabolic-index fibers, and can be found similarly by the present approach for arbitrarily indexed fibers.

One of the most important parameters for the anisotropic fibers is the normalized polarization birefringence defined by [2]

$$
B=\frac{(\beta x-\beta y)}{(\beta x+\beta y) / 2}=\frac{\left(n_{d x}^{2}-n_{d y}^{2}\right) k^{2}+\left(W_{x}^{2}-W_{y}^{2}\right) / a^{2}}{(\beta x+\beta y)^{2} / 2}
$$

In the weakly guidance limit, $\beta_{i}$ in the denominator can be replaced by $k \cdot n_{d i}$ or $k \cdot n_{r i}(i=x$ or $y)$. Employing the relations $W_{x}=F\left(V_{x}\right)$ and $W_{y}=F\left(V_{y}\right)$ and noting that $V_{x}$ is very close to $V_{y},(14)$ can be reduced to a simple formula

$$
B=B_{0}+\left(B_{\infty}-B_{0}\right) \cdot G(V) ; \quad G(V)=F(V) \cdot F^{\prime}(V) / V
$$

where

$$
B_{0}=\frac{\left(n_{d x}-n_{d y}\right)}{\left(n_{d x}+n_{d y}\right) / 2} \quad \text { and } \quad B_{\infty}=\frac{\left(n_{r x}-n_{r y}\right)}{\left(n_{r x}+n_{r y}\right) / 2}
$$

are the normalized birefringence at low and high-frequency limits, respectively. The function $G(V)$, varying from zero at low frequency limit to unity at high frequency limit, defines the frequency dependence of birefringence characteristics. Since the explicit expression for $F(V)$ is available, it becomes straightforward to find the birefringence characteristics for anisotropic fibers with arbitrary index profile.

\section{NumERICAL RESULTS}

For circular fibers with anisotropic core but isotropic cladding, the permittivity model in (12) can be assumed [2]

$$
\begin{gathered}
n_{d x}=n_{d y}=n_{d z}=n_{d} \quad \text { and } \\
\Delta_{x}=A_{x} \Delta, \quad \Delta_{y}=A_{y} \Delta, \quad \Delta_{z}=\Delta .
\end{gathered}
$$

The axial normalized frequency $V_{i}$ now is related to the normalized frequency $V$ by

$$
V_{i}=\sqrt{A_{i}} V, \quad(i=x, y) ; \quad V=\operatorname{kan}_{d} \sqrt{\Delta} .
$$

Then, the normalized propagation constants for both polarizations will be

$$
b_{i} \equiv\left[\left(\beta_{i} / n_{d} k\right)^{2}-1\right] / \Delta=W_{i}^{2} / V^{2}=F^{2}\left(\sqrt{A_{i}} V\right) / V^{2}
$$

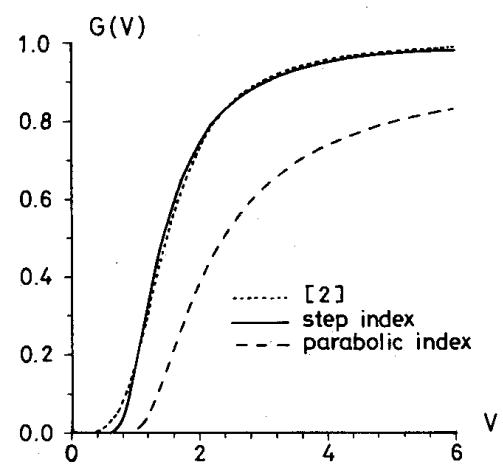

Fig. 1. Birefringence characteristic function $G(V)$ for step and parabolicindex fibers in the limit of weak guidance and perturbational anisotropy.

and the normalized polarization birefringence by (15) becomes

$$
B=\frac{\Delta \cdot q}{2} G(V)
$$

where $q=A_{x} / A_{y}-1$ is the anisotropy parameter.

Fig. 1 shows the birefringence characteristic curve $G(V)$, or $2 B / \Delta q$, for both the step- and parabolic-index fibers. The results for the step-index fiber are in good agreement with those in Fig. 9 of [2] except for smaller $V$, say $V<1.2$. The discrepancy is expected since the functional in [2] does not work well at those frequencies.

For circular fibers with anisotropic core and cladding, one may assume the permittivity model in (12) with [2], [3]

$$
\begin{gathered}
n_{d x}^{2}=A_{x} n_{d}^{2}, \quad n_{d y}^{2}=A_{y} n_{d}^{2}, \quad n_{d z}^{2}=n_{d}^{2} ; \quad \text { and } \\
\Delta_{x}=\Delta_{y}=\Delta_{z}=\Delta .
\end{gathered}
$$

Then, the normalized propagation constant will be given by

$$
\begin{aligned}
b_{i} & \equiv \frac{\left[\left(\beta_{i} / n_{d} k\right)^{2}-1\right]}{\Delta}=\frac{\left(n_{d i} / n_{d}\right)^{2}+\left(W_{i} / n_{d} k a\right)^{2}-1}{\Delta} \\
& =\frac{A_{i}-1}{\Delta}+\frac{1}{V^{2}} \cdot F^{2}\left(\sqrt{A_{i}} V\right) \text { for } i=x \text { or } y
\end{aligned}
$$

The normalized polarization birefringence by (15) now becomes

$$
B=B_{0}=A_{x} / A_{y}-1=q
$$

almost independent of the frequency.

Fig. 2 shows the normalized propagation constant $b_{x}$ versus $V$ for step-index fibers. The parameters are chosen as $A_{x}=$ $1.0002,1.0,0.9998$ and $\Delta=0.004,0.006,0.01$ by fixing $A_{y}=1$. The solid curve gives $b_{x}$ for the isotropic case that $A_{x}=A_{y}=1$. As far as the range with $V>1.2$ is concerned, whence the method in [2] is reliable, the present results are in good agreement with those in Figs. 10 and 11 in [2].

Fig. 3 shows the normalized propagation constant $b_{x}$ versus $V$ for parabolic-index fibers. For the sake of comparison with [3, Fig. 5], the parameter $A_{x}$ is chosen $1.0002,1.00002,1.0$, and 0.9998 by fixing $A_{y}=1.0$, and $\Delta=0.004$. Since the modal field distribution is more complicated in the parabolicindex fibers than those in step-index fibers, it becomes very 


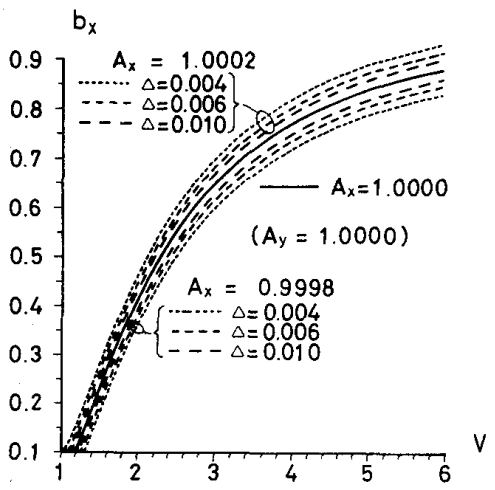

Fig. 2. Dependence of the dispersion relation on the index difference $\Delta=0.004,0.006,0.01$, and the anisotropy parameter $A_{x}=1.0002,1.0$, 0.9998 for step-index fibers.

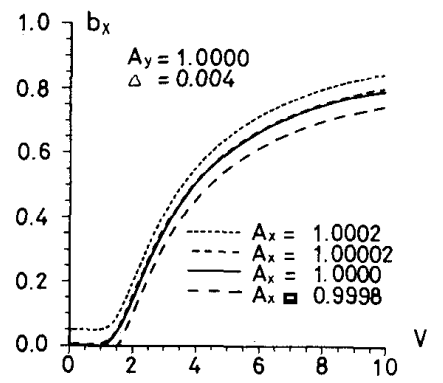

Fig. 3. Dependence of the dispersion relation on the anisotropy parameter $A_{\mathrm{x}}=1.0002,1.00002,1.0$, and 0.9998 for parabolic-index fibers.

difficult to find the suitable trial functions required in the variational analysis [3]. The computed results in [3] are not reliable for near cutoff modes nor for well guided modes. In the midfrequency range with $0.2<b<0.7$, our results can be compared with those in [3] with reasonable agreement.

Finally, consider step-index circular fibers with more general birefringence model. Without loss of generality, assume that $x$ is the principal axis such that $n_{r x} \geq n_{r y}$ and $n_{d x} \geq n_{d y}$. The index difference and the index anisotropy, critical in the propagation characteristics, are defined as

$$
\begin{aligned}
d_{r d} & =1-n_{d x} / n_{r x} \text { and } \quad d_{x y r}=1-n_{r y} / n_{r x}, \\
d_{x y d} & =1-n_{d y} / n_{d x} .
\end{aligned}
$$

Now, let the generalized parameters $V$ and $b_{i}(i=x, y)$ be defined by [5]

$$
V=k a n_{d x} \sqrt{\Delta_{x}} \text { and } b_{i}=\left[\left(\beta_{i} / n_{d x} k\right)^{2}-1\right] / \Delta_{x} .
$$

Then, the normalized $b_{i}$ are

$$
b_{x}=F^{2}(V) / V^{2} \text { and } b_{y}=F^{2}\left(\sqrt{A_{y}} V\right) / V^{2}+b_{y 0}
$$

where

$$
\begin{aligned}
A_{y} & =\frac{n_{r y}^{2}-n_{d y}^{2}}{n_{r x}^{2}-n_{d x}^{2}} \cong \frac{d_{x y d}-d_{x y r}}{d_{r d}}+1 \text { and } \\
b_{y 0} & =-\frac{n_{d x}^{2}-n_{d y}^{2}}{n_{r x}^{2}-n_{d x}^{2}} \cong-\frac{d_{x y d}}{d_{r d}} .
\end{aligned}
$$

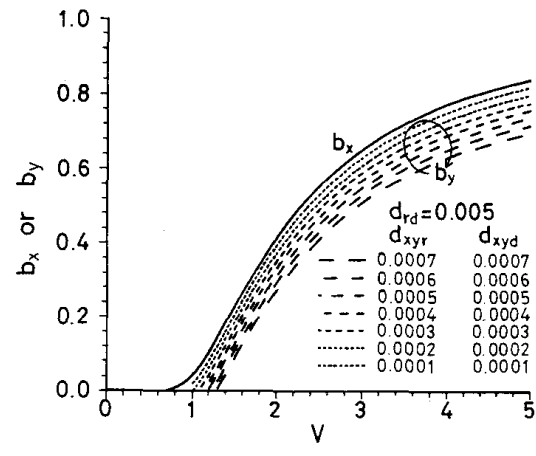

Fig. 4. Dispersion relation of biaxial fibers.

The normalized polarization birefringence is thus given by (15) with parameters

$$
\begin{aligned}
B_{0} & =\frac{d_{x y d}}{1-d_{x y d} / 2} \cong d_{x y d} \text { and } \\
B_{\infty} & =\frac{d_{x y r}}{1-d_{x y r} / 2} \cong d_{x y r} .
\end{aligned}
$$

In all the numerical calculations, $n_{r x}$ is set to 1.5 while other indices are varied by changing $d_{x y r}, d_{x y d}$, and $d_{r d}$. Here, the mode polarized mainly in the $x$-direction is more fundamental, whose normalized propagation constant is denoted by $b_{x}$. Curves of $b_{x}$ versus $V$ for various values of $d_{x y d}$ and $d_{x y r}$ are indistinguishable, as depicted by (21) and shown in Fig. 4. Also shown in the figure are the curves for $b_{y}$ which do vary with $d_{x y d}$ and $d_{x y r}$. This figure can be compared with [5, Fig. 4] which is based on a tedious perturbation analysis. Both are in agreement.

Of the particular interest is the difference $b_{x}-b_{y}$, which is related to the normalized polarization birefringence $B$ defined in (14) by $b_{x}-b_{y} \cong B / d_{r d}$. In Figs. 5-7, the polarization birefringence is plotted as a function of $V$ with $d_{x y d}$ and $d_{x y r}$ as parameters. The results are of the same parameters and can be compared with those in [5, Figs. 5-7]. It should be recalled that the present analysis, with explicit dispersion relation available, is simple and direct. Also, the results for a large frequency range of $0<V<10 \mathrm{can}$ be obtained without any difficulty.

In Fig. $5, d_{x y r} \cong B_{\infty}$ is kept constant while $d_{x y d} \cong B_{0}$ varies. Since the cladding anisotropy is chosen larger than the core anisotropy here, the difference $b_{x}-b_{y}$ decreases from its maximum $d_{x y d} / d_{r d}$ at the low frequency limit and tends to its high-frequency limit $d_{x y r} / d_{r d}$. The behavior can be explained easily in terms of (15). For fibers operating near cutoff, fields spread out to the cladding region such that the cladding anisotropy $d_{x y d}$ should and do have a strong influence on the birefringence characteristics. On the other hand, for fibers operating in the well-guided region, fields are concentrated in the core region such that $d_{x y r}$ is dominant. This is also shown in Fig. 6 where $d_{x y d}$ is kept constant while $d_{x y r}$ varies. In Fig. $7, d_{x y d}=d_{x y r}$ and they vary simultaneously. For these cases, the difference $b_{x}-b_{y}$ is almost independent of $V$. 


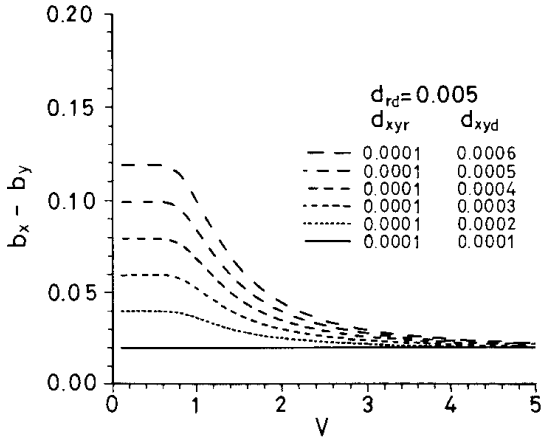

Fig. 5. Birefringence of biaxial fibers with $d_{r d}=0.005$ and $d_{\mathrm{xyr}}=$ 0.0001 .

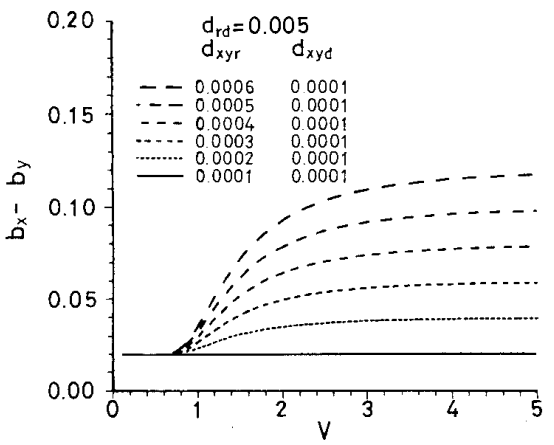

Fig. 6. Birefringence of biaxial fibers with $d_{r d}=0.005$ and $d_{\text {xyd }}=$ 0.0001 .

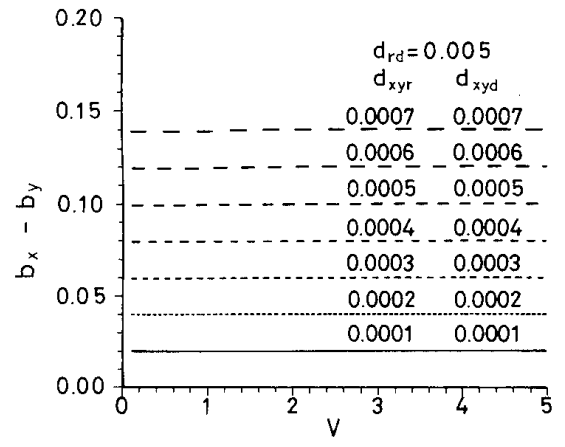

Fig. 7. Birefringence of biaxial fibers with $d_{r d}=0.005$ and $d_{x y r}=d_{x y d}$.

\section{Discussion AND CONCLusions}

A hyper-perturbation theory is applied to find the expansion coefficients of the dispersion relation up to arbitrary orders. After the conventional finite element analysis to search for the eigenvalues, these expansion coefficients are directly calculated by a few simple matrix vector multiplications. Without any numerical differentiation involved, the results of high accuracy can be achieved with few division elements only. With these coefficients, an expansion formula valid for a certain frequency range is available. The explicit dispersion relation, which is very convenient for practical applications, can then be made up from the expansion formulas at several distinct frequencies.

Given the explicit dispersion relation of single-mode optical fibers, the paper presents a simple birefringence analysis for optical fibers with biaxially anisotropic core and/or cladding. The polarization birefringence between the two fundamental modes depends critically on the index difference and the index anisotropy. For fibers operating near cutoff, the cladding anisotropy determines the birefringence characteristics. On the other hand, the core anisotropy is dominant for fibers operating in the well-guided region. For the frequencies in between, the polarization birefringence lies somewhat between the above two limits and is governed by the birefringence characteristic function which can be found from the derivative of the dispersion relation of the corresponding isotropic fibers.

\section{REFERENCES}

[1] I.P. Kaminow, "Polarization in optical fibers," IEEE J. Quantum Electron., vol. QE-17, pp. 15-22, July 1981

[2] I. V. Lindell and M. I. Oksanen, "Transversely anisotropic optical fibers: Variational analysis of a nonstandard eigenproblem," IEEE Trans. Microwave Theory Tech., vol. MTT-31, pp. 736-744, Sept. 1983.

[3] M.I. Oksanen and I. V. Lindell, "Variational analysis of anisotropic graded-index optical fibers," J. Lightwave Technol., vol. 7, pp. 87-91, Jan. 1989.

[4] R. B. Wu and C.H. Chen, "Birefringence analysis of anisotropic optical fibers using variational reaction theory," IEEE Trans. Microwave Theory Tech., vol MTT-34, pp. 741-745, June 1986.

[5] C.L. Chen, "An analysis of high birefringence fibers," J. Lightwave Technol, vol. LT-5, pp. 53-69, Jan. 1987.

[6] R. B. Wu and C. H. Chen, "On the variational reaction theory for dielectric waveguides," IEEE Trans. Microwave Theory Tech., vol. MTT-33, pp. 477-483, June 1985

[7] K.J. Bathe and E.L. Wilson, Numerical Methods in Finite Element Analysis. New Jersey: Prentice-Hall, 1976.

[8] W.L. Mammel and L. G. Cohen, "Numerical prediction of fiber transmission characteristics from arbitrary refractive-index profiles," Appl. Opt., vol. 21, pp. 699-703, 1982.

[9] A. Sharma and S. Banerjee, "Chromatic dispersion in single mode fibers with arbitrary index profiles: A simple method for exact numerical evaluation," J. Lightwave Technol., vol. 7, pp. 1919-1923, Dec. 1989.

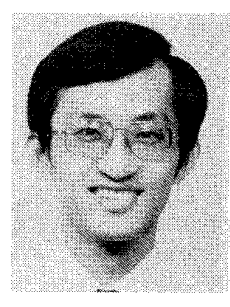

Ruey-Beei Wu was born in Tainan, Taiwan, Republic of China, on October 27, 1957. He received the B.S.E.E. degree from National Taiwan University, Taipei, Taiwan, in 1979, and the Ph.D. degree from the same university in 1985 .

In 1982, he joined the faculty of the Department of Electrical Engineering, National Taiwan University, where he is now a Professor. In 1986, he was a Visiting Scientist for one year in IBM General Technology Division Laboratory, East Fishkill Facility, Hopewell Junction, NY. He works primarily on the applications of numerical methods to electromagnetic field problems. He has been engaged in researches on dielectric waveguides, optical fibers, wave scattering of anisotropic objects, edge slot antennas, microstrip discontinuities, and interconnection modeling for computer packaging. 